\title{
Relevance of Extra Digital Flexion Creases: A Study in North India
}

\section{Dey $S^{*}$ and Kapoor AK}

\author{
Department of Anthropology, University of Delhi, Delhi, India
}

*Corresponding author: Dey S, UGC-JRF, Research Scholar, Department of Anthropology, University of Delhi, Delhi - 110007, India, Tel: 8800853589, E-mail: sangitadey29@gmail.com

Citation: Dey S, Kapoor AK (2017) Relevance of Extra Digital Flexion Creases: A Study in North India. J Forensic Sci Criminol 5(4): 404

Received Date: March 30, 2017 Accepted Date: September 19, 2017 Published Date: September 22, 2017

\begin{abstract}
The proposed study was undertaken among 152 male and 155 female participants belonging to one population group of North India within age range 18-60 years. They were examined for extra digital flexion creases on the volar surface of all digits. Palm prints were obtained from both the right and left hand of each individual. The result showed that out of 3070 digits of 307 participants, 46 digits from 23 ( 16 male and 7 female) participants show the prevalence of extra digital flexion crease either on right or left palm. Ring finger shows the highest percentage of supernumerary volar digital flexion crease among all digits and among both the right (26.09\%) and left (39.13\%) palm. It can be concluded that the supernumerary volar digital flexion creases in humans are a rare and highly individualistic occurrence which can be utilized in concealing the identity of the criminals in forensic investigations. Similar unusual characteristics need to be reported for their rarity and further research remains absolutely necessary to shed light on its predictive power. It has great implications in global humanitarian, medico-legal missions and in cases where digital images or CCTV footage of the hand were recovered and brought for examination to identify the potential suspects which ultimately aids in criminal authentication and technological advancement.
\end{abstract}

Keywords: Digital; Flexion crease; Volar surface; Extra; Forensic investigation

\section{Introduction}

Flexion creases are defined as the characteristics present transversely in between the dermatoglyphic ridges as grooves on the volar (or palmar) surface of the each finger at the vicinity of the inter-phalangeal joint [1]. They indicate the location of firmer attachment of the skin to the underlying structures and correspond to the regions where accelerated growth does not take place [2]. Due to this strong attachment to the underlying structures, it provides ease of the movement of the digits and stability required for strong grasping. These flexion creases are the unique features of the hand. These are also considered as one of the external anatomical landmarks of the hand in addition to palmar, thenar and wrist creases. Each hand has twenty-seven bones and out of which fourteen belongs to phalanges or finger bones and each finger has three phalanges- the proximal, intermediate and the distal whereas thumb has only two phalanges - the proximal and the distal [3]. Each phalanx corresponds to the area where digital flexion crease occurs. Three digital flexion creases are normally observed to exist on the volar surface of each finger of the hand except for the thumb - distal inter-phalangeal crease, proximal inter-phalangeal crease and metacarpo-phalangeal crease except thumb because of its different anatomy [4]. Additional or extra creases was defined for the first time by Dejong and Platou (1967) as creases present in addition with the usual inter-phalangeal crease which disrupts the local ridge configuration [5]. Extra creases are easily identified as those creases which are separated from the inter-phalangeal crease by two or more epidermal ridges.

Volar digital transverse creases are not caused primarily by embryonic flexion movements $[1,6]$. The origin or timing of appearance digital flexion creases is based on some controversy and disagreement. The first hypothesis is that digital flexion creases develop secondary to flexion movements of the developing hand. However, studies conducted by Kimura \& Kitagawa demonstrated that digital flexion creases begin to appear between 7 and 9 weeks of gestation age while spontaneous flexion or movement of the digits does not occur until 11.5 weeks of gestation [7]. Thus, the hypothesis was refuted that the flexion creases arise secondary to functional movement of the digits and hand [7,8]. It is concluded that these flexion creases develop during early fetal life. The second hypothesis is that the flexion creases arise independent of fetal palm movement and are genetically determined [7]. The incidence of extra, displaced and missing volar digital flexion creases in individuals without any radiographic anomalies in the anatomy of the hand joints and unusual volar digital transverse creases patterns in a number of syndromes indicates that though genetic factors do probably play a role in digital creases development, fetal hand movement is necessary for normal creases pattern formation $[8,9]$. 
Different patterns of digital flexion are found associated with a number of genetic syndromes. Excessive or increased number of volar digital flexion creases had been reported in Allagile syndrome, partial deletions of chromosome 1q, fetal alcohol syndrome, Cerebro-oculo-facio-skeletal syndrome as well as in Sickle cell anemia [8,10-13]. A reduced number of digital flexion creases has been described to be also associated with disorders such as a single inter-phalangeal crease on the little finger represents a characteristic dermatoglyphic trait in Down's syndrome, 18-trisomy, partial trisomy 9p, de Lange syndrome [14-19]. Missing distal interphalangeal flexion creases are encountered in a mentally retarded individual [1]. Both extra or supernumerary and reduced number of flexion creases can be found in patients with Larsen syndrome [20]. However, literature reported that single bilateral and extra inter-digital flexion crease of the fifth finger can be found in phenotypically normal male and female without any abnormalities of the bones and joints or evidence of dyskinesia [8,21]. Unusual flexion creases are also encountered in normal healthy individuals $[4,15,22]$. But limited numbers of studies are conducted on this front. Considering the clinical syndromes associated with digital flexion creases and due to the paucity of the literature on the issue, it becomes reasonable to conduct this preliminary study which aims at documenting the incidence or prevalence patterns of extra volar digital flexion creases in normal healthy individuals for application in the forensic domain.

\section{Materials and Methods}

The proposed cross sectional survey study was undertaken among participants belonging to one ethnic group of North India. This study was conducted in Dhikauli village in Pilana Tehsil under district Baghpat of Uttar Pradesh state, India. Jat population group within age range 18-60 years were randomly selected to conduct the research study. Participants without any medical history of hand, disease, injury, deformity, abnormality, genetic disorder were included in the research study. Each participant was required to fill in a questionnaire containing basic demographic profile such as age; sex; ethnicity. Ethical clearance was taken from the ethical committee of the Department of Anthropology, University of Delhi. Informed consent was granted from all participants involved in the study. They were made properly aware about the objectives, relevance, and purpose of the study and were assured about the confidentiality of the data.

The palm print was obtained from both the right and left hand as suggested by Cummins \& Mildo [23]. A small amount of printing ink was taken on a glass slab and is spread uniformly over the glass slab using the cotton pad till a thin film is obtained. By using this cotton pad, the ink was distributed evenly through the finger in equal proportion. The area of metacarpo-phalangeal crease was given special attention. The sheet of paper is kept over the palmar pad and the proximal part of the palm is brought in contact with the paper first, followed by the distal part. In order to ensure printing of the creases, the pressure is everted particularly over the metacarpo-phalangeal region of the hands. Then the palm is slowly lifted radio - ulnar wards carefully to avoid any smudging. Palmar Prints which clearly revealed distal, proximal and metacarpo-phalangeal crease were used to analyze extra digital flexion crease.

A total of 3070 digits of 152 males and 155 females were studied and incidence of digital flexion crease on both right and left palms was depicted in a suitably designed data form. All the digits in the palm were thoroughly examined and documented for the prevalence of supernumerary digital flexion creases. Hand images were also obtained using digital camera in cases were extra digital flexion creases was encountered. Data was analyzed using SPSS Statistical Package for Social Sciences version 17 and simple descriptive statistics was performed. The flexion creases are qualitative in nature, and thus only the frequencies were computed to satisfy the objectives of the study and only those differences which are at a significance level of $\alpha=0.01$ were regarded as significant.

\section{Results}

The basic demographic profile of the participants and incidence rate of extra digital flexion crease was depicted in Table 1. Out of 152 male (49.51\%) and 155 female (50.49\%) participants, 23 (1 in 13) participants were found to show extra digital flexion crease $(7.49 \%)$. And out of that 23 participants who have extra digital flexion crease, 16 were males and 7 were females. Thus, the occurrence of supernumerary volar digital flexion crease was also more prevalent in males than females (ratio 16:7).

\begin{tabular}{|c|c|c|c|}
\hline \multicolumn{2}{|c|}{ Profile } & Number & Percentage \\
\hline \multirow{2}{*}{ Sex } & Male & 152 & 49.51 \\
\cline { 2 - 4 } & Female & 155 & 50.49 \\
\hline \multirow{2}{*}{$\begin{array}{c}\text { Incidence of } \\
\text { E-DFC }\end{array}$} & Absent & 284 & 92.51 \\
\cline { 2 - 4 } & Present & 23 & 7.49 \\
\hline
\end{tabular}

Table 1: Demographic profile of participants and incidence of extra digital flexion crease (E-DFC)

Table 2 shows the prevalence or incidence rate of digital flexion creases on the right and left hand. The result showed that left hand represents an increased percentage (25.22\%) of extra volar digital flexion creases than the right hand (14.78\%). Ring finger shows the highest percentage of extra digital flexion crease among all digits and among both the right (26.09\%) and left (39.13\%) hand. Of particular interest, it can be observed that extra digital flexion crease are absent on thumb in the studied population. Figure 1,2,3,4 and 5 represents the pictures of participant's hand with extra digital flexion creases. It can be suggested from the pictures that the 
occurrence of extra digital flexion crease is not restricted to occur in any particular finger. Similar results were depicted among Nigerians that the incidence of the extra digital crease was more prevalent among the males than the females (ratio 5:3) in the left than the right and in the ring finger [24].

\begin{tabular}{|c|c|c|c|c|c|c|c|c|c|c|}
\hline \multirow{2}{*}{ Subject } & \multicolumn{5}{|c|}{ SNDFC on Right Palm } & \multicolumn{5}{|c|}{ SNDFC on left Palm } \\
\hline & Thumb & Index & Middle & Ring & Little & Thumb & Index & Middle & Ring & Little \\
\hline 1 & - & - & - & - & + & - & - & - & - & + \\
\hline 2 & - & - & - & + & - & - & - & - & - & - \\
\hline 3 & - & - & - & - & + & - & - & - & - & - \\
\hline 4 & - & - & - & - & - & - & - & + & - & + \\
\hline 5 & - & - & - & - & - & - & + & + & + & - \\
\hline 6 & - & + & + & - & - & - & - & - & - & - \\
\hline 7 & - & - & - & + & - & - & - & - & + & - \\
\hline 8 & - & - & - & - & - & - & - & + & + & - \\
\hline 9 & - & - & - & - & - & - & + & + & + & + \\
\hline 10 & - & - & + & - & - & - & - & - & - & - \\
\hline 11 & - & - & - & + & - & - & - & - & - & - \\
\hline 12 & - & + & + & - & - & - & - & - & - & - \\
\hline 13 & - & + & - & - & - & - & - & + & + & - \\
\hline 14 & - & - & - & - & - & - & + & + & + & + \\
\hline 15 & - & - & - & + & - & - & - & - & - & + \\
\hline 16 & - & - & + & + & - & - & - & - & - & - \\
\hline 17 & - & + & - & + & - & - & - & - & - & - \\
\hline 18 & - & - & - & - & - & - & + & - & + & - \\
\hline 19 & - & - & - & - & - & - & - & + & + & - \\
\hline 20 & - & - & - & - & + & - & - & - & - & - \\
\hline 21 & - & - & - & - & - & - & - & - & - & + \\
\hline 22 & - & - & - & - & - & - & - & + & - & + \\
\hline 23 & - & - & - & - & - & - & + & - & + & - \\
\hline Total & 0 & 4 & 4 & 6 & 3 & 0 & 5 & 8 & 9 & 7 \\
\hline$\%$ on digits & 0 & 17.39 & 17.39 & 26.09 & 13.04 & 0 & 21.74 & 34.78 & 39.13 & 30.43 \\
\hline$\%$ on Palm & \multicolumn{5}{|c|}{14.782} & \multicolumn{5}{|c|}{25.216} \\
\hline
\end{tabular}

Table 2: Incidence rate of extra digital flexion crease (E-DFC) on the right and left hand

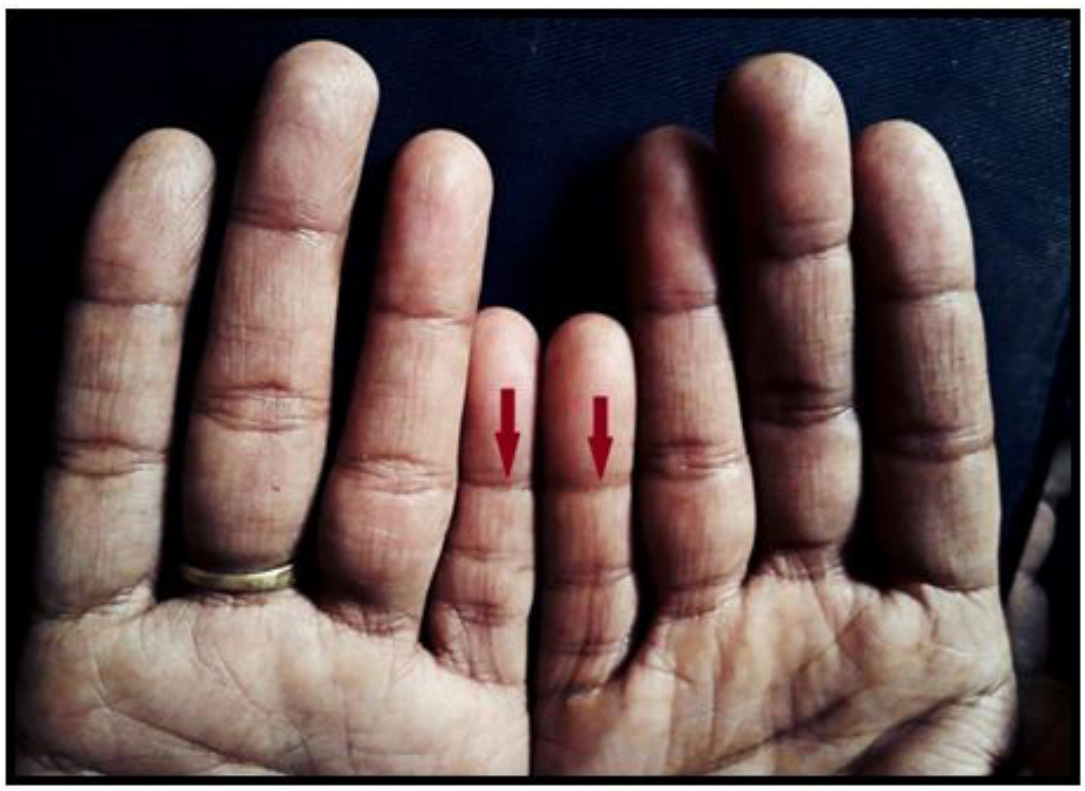

Figure 1: Extra digital flexion crease present on little finger bilaterally 


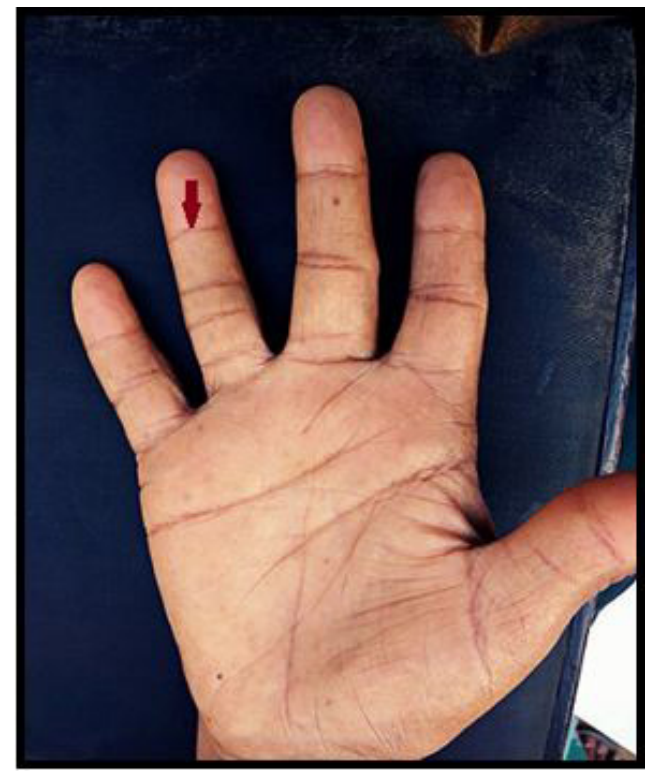

Figure 2: Right palm showing extra digital flexion crease on ring finger

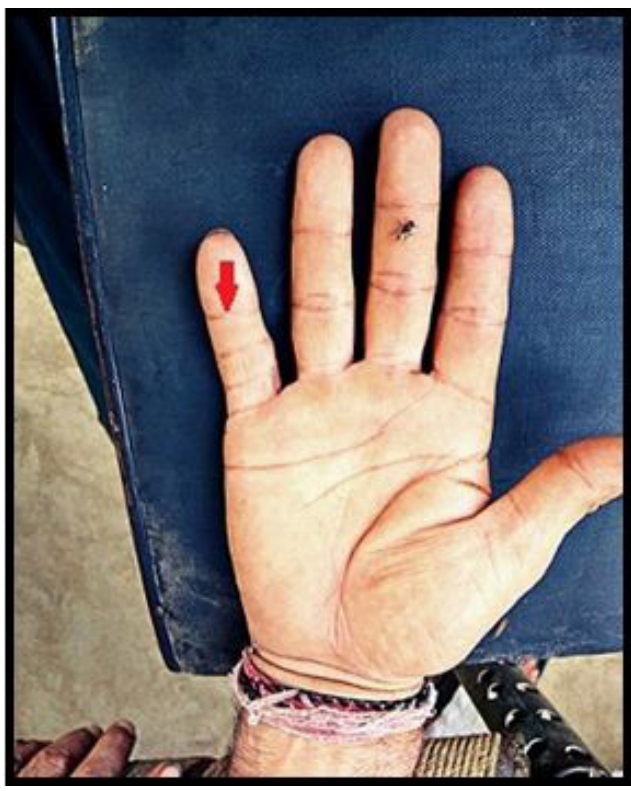

Figure 3: Right palm showing extra digital flexion crease on little finger (Unilaterally)

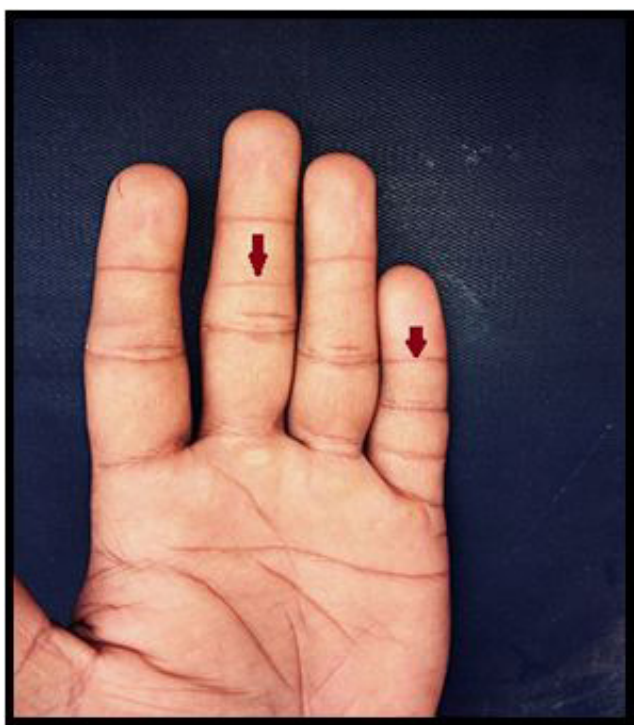

Figure 4: Left palm showing extra digital flexion crease on middle and little finger 


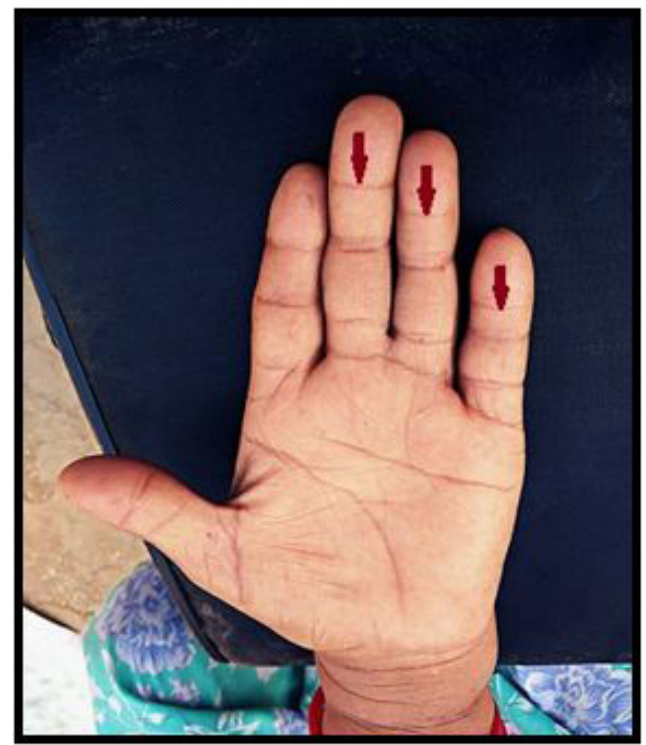

Figure 5: Left palm showing extra digital flexion crease on middle, ring and little finger

\section{Discussion}

In the present study, about $1.5 \%$ of the digits show the prevalence of supernumerary or extra digital flexion creases either on right or left palm in normal individuals. However, there are investigations which reveal that unusual flexion creases are strongly associated with chromosomal abnormalities and genetic disorder $[8,10,13]$. Thus one can imagine how these participants without any disorder would have grappled with the stated conditions. The radiological examination in the previous study confirmed the normal number of phalanges and no skeletal deformity in the respective finger which possess unusual flexion crease [4,21,25]. However, the causes behind such a rare characteristic are yet to be revealed and need proper investigation but it is indicated that extra digital creases point's towards genetic determinants which play a role in crease occurrence. Researchers are trying to elucidate this issue by using animal models as it may be proven beneficial for syndrome identification, in the identification and subsequent prosecution of paedophilic sex crimes [26,27]. Also, the occurrence of excessive digital flexion creases follows a multi-digital fashion and not restricted to occur in any particular digit and can occur in any digit [28]. Thus, it can be suggested that extra digital flexion crease is extremely rare finding in normal healthy individuals.

Forensic experts often involved in global humanitarian and medico-legal missions to conceal forensic identification of unknown dismembered and fragmentary remains recovered from mass disasters, explosions or any other incidents. During the forensic examination, the main aim of the forensic experts is to estimate age, sex, ancestry, and stature. In such cases, peculiar, rare and unique characteristics such as supernumerary or extra digital flexion creases may help in establishing the identity of the decreased by comparing with the antemortem records. Many studies have reported that the peculiar features of the hand such as scars, moles, birthmarks, cuts, tattoos may act as individualistic characteristics and thus can assist in the forensic identification of the criminals and victims in cases where faces of the criminals could not be recovered but digital images or CCTV footage of the hand from the crime scene were commonly recovered and brought for forensic examination. Similarly, the extra digital flexion creases may also prove to be strong forensic evidence in such cases.

It can be concluded that the extra digital flexion creases in humans are a rare and highly individualistic occurrence which can be utilized in concealing the identity of the criminals in forensic investigations. However, the characteristic need to be investigated and studied thoroughly in varied population groups and further research remain absolutely necessary in this direction so that the exact reason for the incidence and occurrence of the extra digital flexion creases may be known in different ethnic groups. Similar unusual characteristics need to be reported and published for their rarity and significance in forensic medico-legal cases and criminal authentication which ultimately aids in the advancement of science and technology.

\section{Acknowledgement}

SD was thankful to UGC for financial support under which the field work was conducted. Thanks are also due to the subjects of district Baghpat in Uttar Pradesh for their help and coordination for data collection.

\section{References}

1. Aue-Hauser G (1979) Flexion creases on fingers. In Wertelecki W, Plato CC. (eds) Dermatoglyphics-Fifty Years Later. Alan R Liss, Inc., New York.

2. Singh I (2002) The phanlenges of the hand, bones of the upper limb. Essentials of Anatomy. Jaypee Brothers Medical Publishers, New Delhi 17.

3. Aue-Hauser G, Okajima M, Wytek R (1980) Digital Flexion Creases in Japanese. J Anthropol Soc Nippon 88: 443-54.

4. Komatz Y, Daijo K, Yoshida O (1978) Extra interphalangeal transverse creases of the little finger. Jinrui Idengaku Zasshi 23: 31-6. 
5. DeJong R, Platou RV (1967) Sickle cell hemoglobinopathy. An anatomic sign. Am J Dis Child 113: 271-2.

6. Okajima M (1966) A dermatoglyphical study of metacarpophalangeal creases. Am J Phys Anthropol 24: 371-80.

7. Kimura S, Kitagawa T (1986) Embryological development of human palmar, plantar, and digital flexion creases. Anat Rec 216: $191-7$.

8. Kamath BM, Loomes KM, Oakey RJ, Krantz ID (2002) Supernumerary digital flexion creases: an additional clinical manifestation of Alagille syndrome. Am J Med Genet 112: 171-5.

9. Stevens CA, Carey JC, Shah M, Bagley GP (1988) Development of human palmar and digital flexion creases. J Pediatr 113: 128-32.

10. Watson MS, Gargus JJ, Blakemore KJ, Katz SN, Breg WR (1986) Chromosome deletion 1q42-43. Am J Med Genet 24: 1-6.

11. Jones KL, Smith DW (1973) Recognition of the fetal alcohol syndrome in early infancy. Lancet 302: 999-1001.

12. Lurie IW, Cherstvoy ED, Lazjuk GI, Nedzved MK, Usoev SS (1976) Further evidence for the autosomal-recessive inheritance of the COFS syndrome. Clin Genet 10: 343-6.

13. Zizmor J (1973) The extra transverse digital crease: a skin sign found in sickle cell disease. Cutis 11: 447-9.

14. Penrose LS (1931) The creases on the minimal digit in mongolism. Lancet 218: 585-6.

15. Plato CC, Cereghino J, Steinberg FS (1973) Palmar dermatoglyphics of Down's syndrome: Revisited. Pediatr Res 7: 111-8.

16. Schaumann B, Alter M (1976) Dermatoglyphics in Medical Disorders. Springer Verlag, New York.

17. Hodes ME, Cole J, Palmer CG, Reed T (1978) Clinical experience with trisomies 18 and 13. J Med Genet 15: 48-60.

18. Rodewald A (1979) Dermatoglyphics in partial trisomy 9p + and in the partial monosomy 9p-. - Type and contretypell hypothesis. Bratislava: $8^{\text {th }}$ Bartos's Symp Dermatoglyphics.

19. Preus M, Fraser FC (1972) Dermatoglyphics and syndromes. Amer J Dis Child 124: 933-43.

20. Dallapiccola B, Capra L (1973) Dermatoglyphics in Larsen's syndrome. Lancet 301: 493-4.

21. Mestrovic T, Ozegic O (2012) Single bilateral interdigital flexion crease of the fifth finger in a phenotypically normal male. Int J Anat Var 5: 113-5.

22. Alter M (1970) Variation in palmar creases. Am J Dis Child 120: 424-31.

23. Cummins H, Mildo C (1926) Palmar and plantar epidermal ridge configurations (dermatoglyphics $\dagger$ ) in European-Americans. Am J Phys Anthrop 9: 471-502.

24. Uko VA, Nwaopara AO, Olugbenga MA, Oyadonghan GP, Imah P, et al. (2012) The incidence and pattern of supernumerary digital flexion creases amongst the population of Ekpoma in Edo, Nigeria. Internat J Basic Appl Innov Res 1: 57-62.

25. Singh B, Krishan K, Kanchan T (2015) Extra phalangeal crease - A trait in forensic identification. J Forensic Leg Med 35: 1-3.

26. Mestrovic T, Ozegic O (2017) Variations of interdigital flexion creases - inciting the curiosity of the researchers, professionals and the public. Int J Anat Var 10: 28-9.

27. Gilchrist R (2010) Forensic human identification of the livingbased upon the palmar flexion creases of the hand. Axis 2: 32-3.

28. Kosztolanyi G, Mehes K (2003) Supernumerary digital flexion crease. Am J Med Genet 121: 90-1.

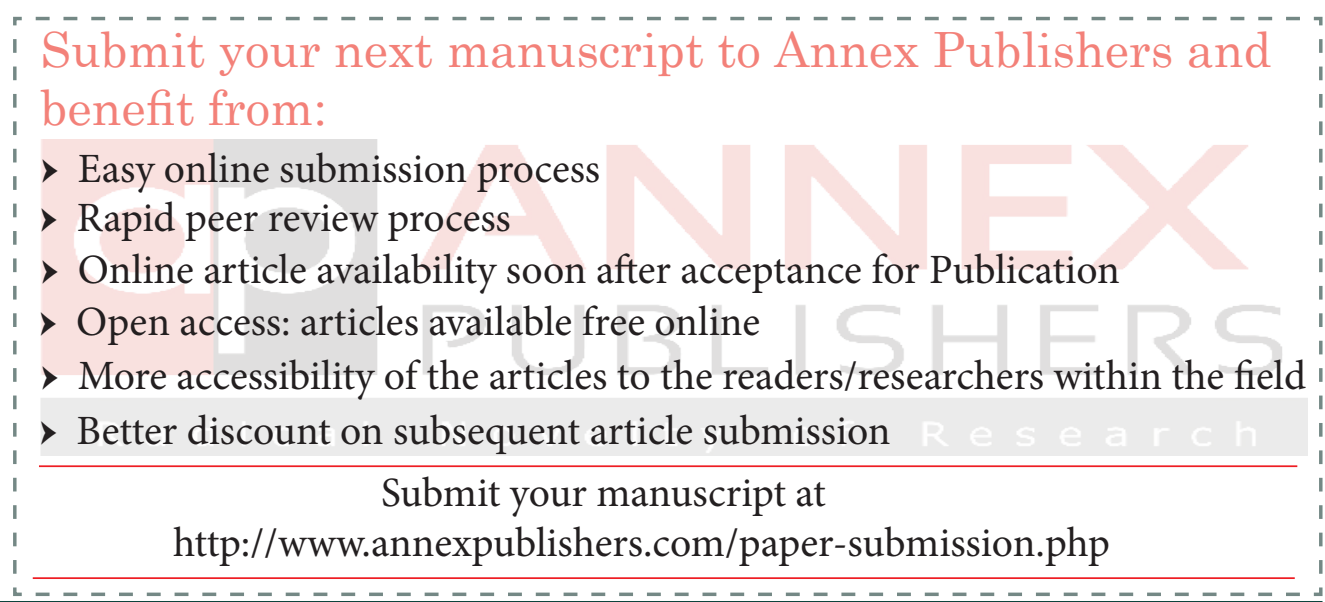

